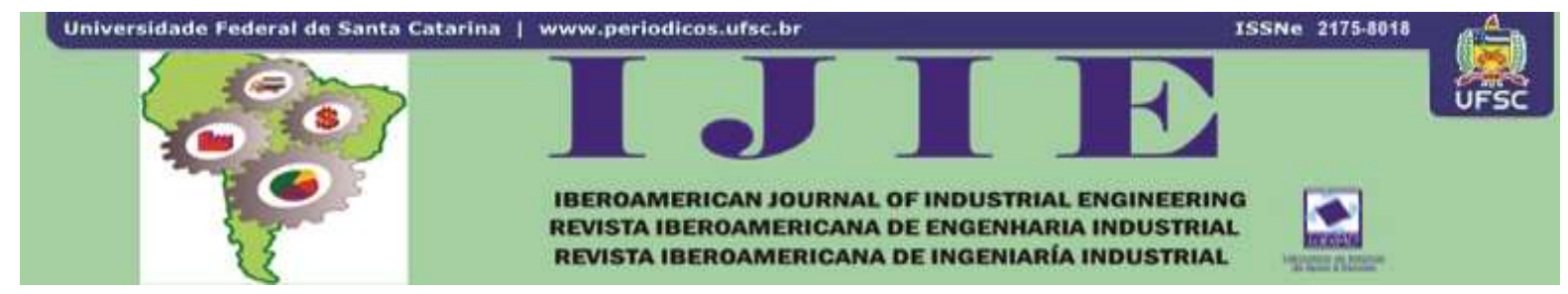

\title{
OPTIMIZACIÓN DE LA PRODUCCIÓN DE POLI ( $\beta$ HIDROXIBUTIRATO) A PARTIR DE GLICEROL
}

\author{
Fernando Daniel Ramos ${ }^{1}$ \\ Vanina Estrada ${ }^{2}$ \\ Maria Soledad Diaz ${ }^{3}$ \\ Marcelo Armando Villar ${ }^{4}$
}

RESUMEN: En este trabajo se ha realizado el diseño preliminar de una planta de producción de poli( $\beta$-hidroxibutirato), PHB, mediante el empleo de microorganismos y a partir de glicerol como materia prima. El proceso consta de tres etapas: purificación de la materia prima, donde la corriente de glicerol, subproducto en la producción de biodiesel, se despoja del metanol que pudiese contener; fermentación, donde se produce PHB a partir de Cupriavidus necátor y por último, extracción del producto, donde se produce la lisis celular y se logra la separación y purificación del biopolímero.

Palabras clave: Cupriavidus necátor. Optimización. Poli( $\beta$-hidroxibutirato).

\section{INTRODUCCIÓN}

La producción de poli(hidroxialcanoatos) mediante microorganismos constituye una alternativa a la producción de polímeros a partir de combustibles fósiles. Asimismo, la producción actual de poli( $\beta$-hidroxibutirato), PHB, a gran escala utiliza sustratos que resultan costosos y/o que compiten con la alimentación humana. En estos procesos, el costo de la materia prima alcanza hasta un $50 \%$ del costo de producción. Consecuentemente, es importante la utilización de fuentes de carbono más económicas, tales como glicerol, que es el

\footnotetext{
1 Ingeniero Químico, Planta Piloto de Ingeniería Química (PLAPIQUI), Consejo Nacional de Investigaciones Científicas y Técnicas (CONICET-UNS), Departamento de Ingeniería Química, Argentina. E-mail: fdramos@plapiqui.edu.ar.

2 Doctora en Biología, Planta Piloto de Ingeniería Química (PLAPIQUI), Consejo Nacional de Investigaciones Científicas y Técnicas (CONICET-UNS), Departamento de Ingeniería Química, Argentina. Email: vestrada@plapiqui.edu.ar.

${ }^{3}$ Doctora en Ingeniería Química, Planta Piloto de Ingeniería Química (PLAPIQUI), Consejo Nacional de Investigaciones Científicas y Técnicas (CONICET-UNS), Departamento de Ingeniería Química, Argentina. Email:sdiaz@plapiqui.edu.ar.

${ }^{4}$ Doctor en Ingeniería Química, Planta Piloto de Ingeniería Química (PLAPIQUI), Consejo Nacional de Investigaciones Científicas y Técnicas (CONICET-UNS), Departamento de Ingeniería Química, Argentina. Email:mvillar@plapiqui.edu.ar.
}

Iberoamerican Journal of Industrial Engineering, Florianópolis, SC, Brasil, v. 6, n. 11, p. 261-270, 2014. 
principal subproducto de la producción de biodiesel.

En Argentina, la industria del biodiesel ha crecido un $2250 \%$ desde el año 2006 (CÁMARA ARGENTINA DE RECURSOS RENOVABLES, 2012), convirtiéndolo en el primer exportador mundial de este biocombustible, con una capacidad de producción instalada al 2012 de 3200000 tn/año. El biodiesel es producido principalmente a partir de la transesterificación de aceites vegetales con Metanol (alcohol de cadena corta) catalizada por un ácido o una base dando como producto secundario el glicerol. Aunque este compuesto en estado puro es utilizado en diversas industrias tales como la farmacéutica, alimenticia, cosmética, etc., la sobreproducción acoplada a la producción de biodiesel hace que la oferta de glicerol sea mayor que la demanda.

El objetivo de este estudio es desarrollar un modelo para el diseño óptimo de una planta de producción de PHB mediante el empleo de microorganismos y a partir de glicerol como materia prima. Para ello, se implementa dicho proceso en el simulador comercial Aspen Plus y se utilizan herramientas de optimización para alcanzar el objetivo planteado. El proceso consta de tres sectores: purificación de la materia prima (glicerol), fermentación y extracción del producto (PHB).

En la etapa de purificación de glicerol, se realiza una optimización de cada uno de los equipos involucrados con el objetivo de obtener un mayor caudal de producto, teniendo en cuenta especificaciones de pureza, con los menores requerimientos de corrientes de servicio (agua de enfriamiento y vapor). Previamente, se efectúa un análisis de sensibilidad de los equipos más complejos para determinar los parámetros más influyentes.

\section{DESCRIPCIÓN DEL PROCESO}

En el proceso de producción de PHB a partir de glicerol se pueden distinguir tres sectores principales en donde se lleva a cabo la purificación del glicerol, fermentación y extracción del producto. Debido a análisis previos de productividad de diferentes cepas de microorganismos, se escogió el Cupriavidus necator para ser inoculado y utilizarlo como productor del biopolímero de interés (POSADA et al., 2011).

\subsection{Sector de Purificación de Glicerol}

Una corriente de glicerol con metanol, agua y restos de triglicéridos, dependiendo de la procedencia de la materia prima (POSADA; CARDONA; RINCÓN, 2010), ingresa a este primer sector, donde se calienta para separar el metanol y parte del agua que ingresa con la 
misma a través de una evaporación instantánea. A continuación, se neutraliza con ácido clorhídrico $(\mathrm{HCl})$ la corriente líquida proveniente del flash en un reactor, previo enfriamiento, con el objetivo de neutralizar el catalizador no utilizado proveniente del proceso previo de producción de biodiesel.

Luego de realizada la reacción de neutralización (Figura 1), se eliminan los sólidos provenientes de la transesterificación (producción de biodiesel) como $\mathrm{NaOCH}_{3}$ y cenizas con la ayuda de una centrifuga. La corriente libre de sólidos insolubles se enfría haciéndola circular por un nuevo intercambiador para el ingreso de la misma a un decantador donde se lava la corriente con agua de proceso para obtener glicerol libre de sales y sólidos.

Finalmente, el agua y las remanentes cantidades de metanol se remueven por evaporación en un flash y una columna de destilación, previo calentamiento a través de otro intercambiador de calor para obtener como producto final de esta primera etapa de proceso una corriente de glicerol la cual posteriormente se diluye para alcanzar las especificaciones necesarias y de esta manera poder brindar a los microorganismos la fuente de carbono necesaria en su crecimiento.

Figura 1 - Esquema del proceso de purificación de glicerol. HE: Intercambiadores de calor, FH: flashes, RC: reactor de neutralización, CT: centrifuga, DC: decantador, CD: columna de destilación

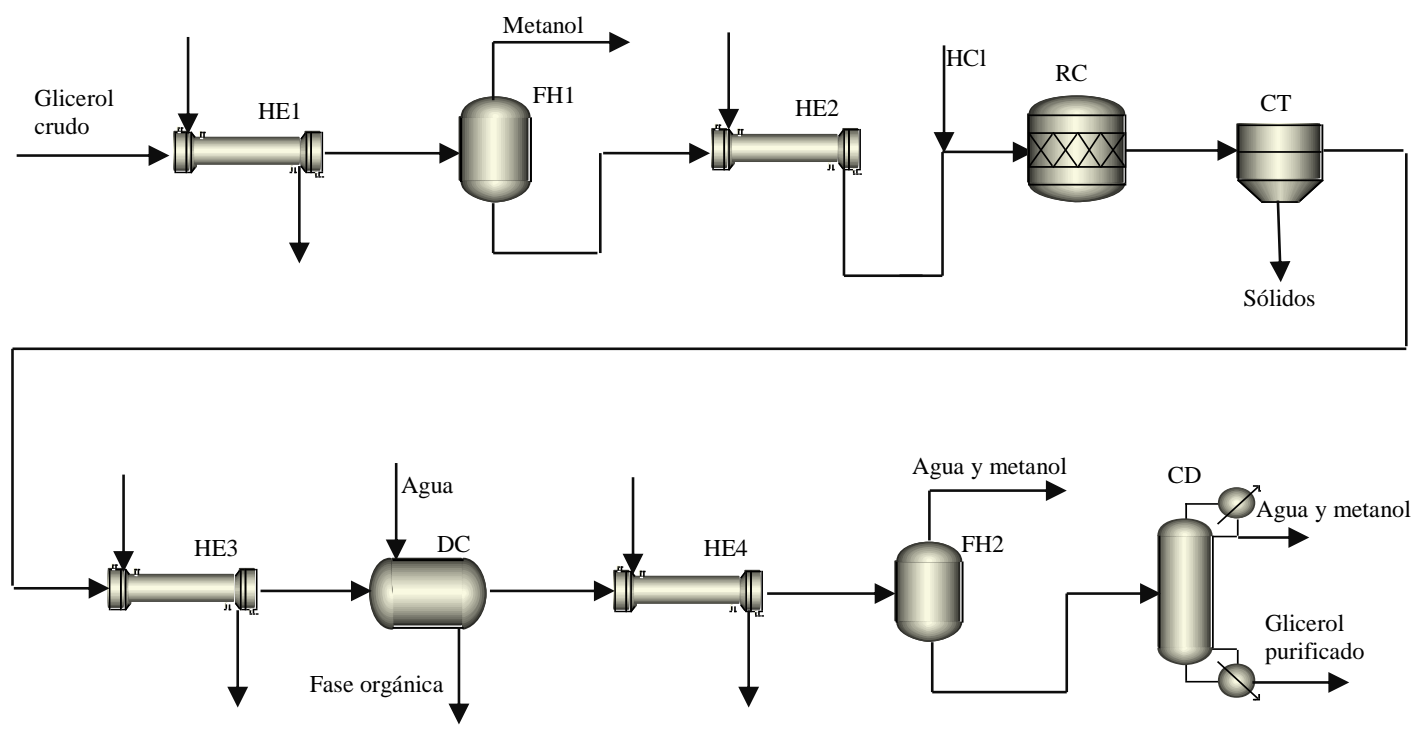

\subsection{Sector de Fermentación}

La corriente de glicerol purificada previamente, se diluye debido a requerimientos del microorganismo y por lo tanto se debe someter a un proceso de esterilización, previo al ingreso al sector de fermentación. Para ello se comprime y calienta la corriente que posee la fuente de carbono que se va a alimentar al medio de cultivo. 
Previo al ingreso del glicerol al primer fermentador se lleva la corriente a condiciones de presión y temperatura ambiente, haciéndola pasar por una válvula y un intercambiador.

En el primer fermentador (Figura 2), se lleva a cabo el crecimiento bacteriano, mientras que en el segundo se produce la acumulación de PHB en los microorganismos luego de someterlos a una limitación de alguno de sus nutrientes esenciales como nitrógeno, fósforo, magnesio, potasio o azufre.

Figura 2 - Esquema del proceso de fermentación.BM: bomba, HE: intercambiadores de calor, VL: válvula, FR: fermentadores

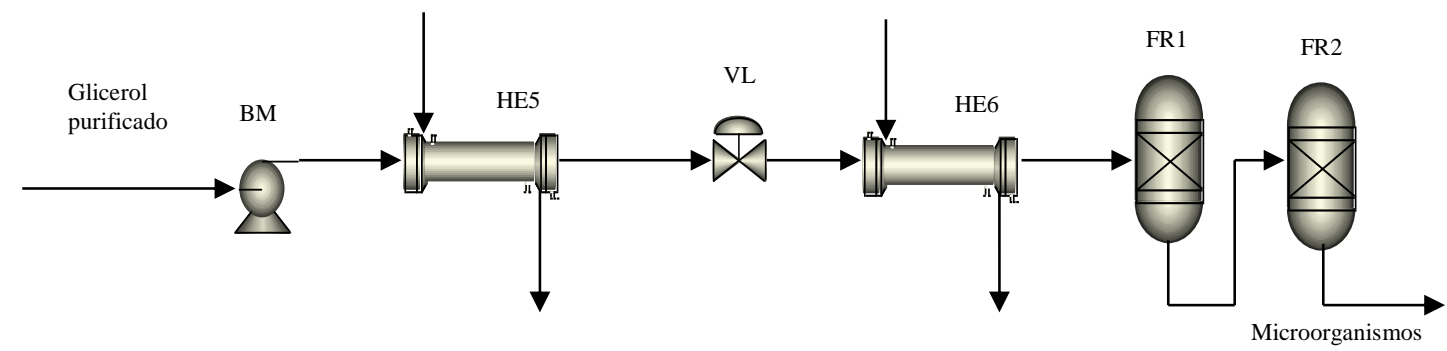

\subsection{Sector de Extracción y Purificación de PHB}

La corriente proveniente del proceso de fermentación, se mezcla con formato de metilo, luego de un calentamiento por un intercambiador, se ingresa a un digestor donde se rompen las paredes celulares para liberar el PHB (Figura 3).

Con una centrífuga se eliminan los restos insolubles de las paredes celulares que se generaron. La corriente rica en producto, agua y solvente se envía a un flash, donde previamente se la calienta por medio de un intercambiador logrando de esta manera la separación y obtención del biopolímero purificado.

Figura 3 - Esquema del proceso de extracción de PHB. HE: intercambiadores de calor, DG: digestor, CT: centrífuga, FH: flash

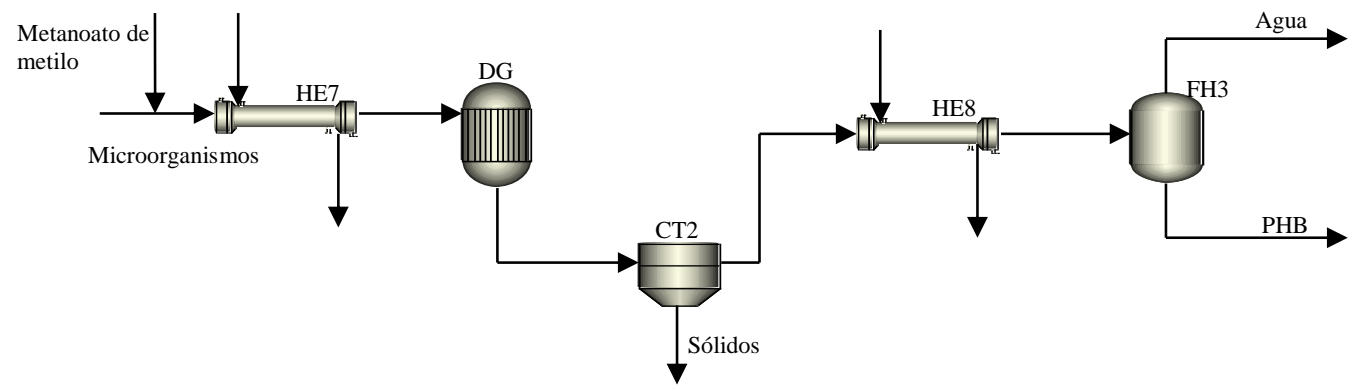

\section{MODELAMIENTO Y OPTIMIZACIÓN}

Los procesos descriptos se han implementado en el simulador comercial ASPEN, para una producción de PHB de 200 tn/año. Se lleva a cabo la optimización de uno de los sectores 
de la planta de producción de PHB, específicamente el correspondiente a la purificación de glicerol, donde se busca maximizar el caudal de materia prima purificado con el fin de lograr un mayor beneficio en la utilización de este subproducto de la industria del biodiesel

Se formula un problema de optimización. A continuación se muestra la representación matemática del mismo:

$$
\begin{gathered}
\operatorname{Min} Z=f(x) \\
\text { s.t } \\
g(x, y) \leq 0 \\
h(x, y)=0 \\
x^{L} \leq x \leq x^{U}
\end{gathered}
$$

Donde $\mathrm{Z}$ es la función objetivo que se busca maximizar, para lograr finalmente obtener en este sector el mayor caudal de glicerol purificado con los menores costos operativos. Esto último se traduce en la minimización de los caudales de corrientes auxiliares para el proceso, como así también en las dimensiones de equipos; $\mathrm{h}(\mathrm{x}, \mathrm{y})$ es el modelo matemático del proceso que se está optimizando, el cual incluye modelos rigurosos de columnas de destilación, tanques flash, intercambiadores de calor, etc.; $\mathrm{g}(\mathrm{x}, \mathrm{y})$ corresponde a restricciones operativas y de calidad de producto, que incluyen recuperación de glicerol en cada una de las etapas de separación, la pureza del glicerol a la salida del sector de purificación, así como diferencias mínimas de temperaturas entre corrientes en los intercambiadores de calor.

Las variables de optimización, $\mathrm{x}$ e y, incluyen temperaturas de operación de los separadores flash, presión de operación de las columnas de destilación, y caudales de las corrientes de servicios. Las predicciones termodinámicas se llevan a cabo con un modelo de coeficiente de actividad para la fase líquida (NRTL) y una ecuación de estado cúbica (RKSoave) para la fase vapor. Dicho modelo provee una representación confiable del equilibrio de fases y propiedades termodinámicas. Finalmente, se calculan las dimensiones de cada uno de los equipos, sobredimensionándolos con un factor de 1.4.

\section{RESULTADOS Y DISCUSIONES}

Como primera etapa, se lleva a cabo un análisis de sensibilidad, con el objeto de determinar las principales variables de optimización del proceso. Este estudio, que considera el impacto que tienen sobre la solución del problema presentado los cambios en los 
parámetros del modelo, se realiza en ASPEN para las distintas unidades del proceso. Se considera la variación de cada uno de ellos de forma individual y manteniendo todos los otros constantes.

Los resultados obtenidos para la torre de destilación se muestran en la Figura 4 y la Figura 5.

Figura 4 - Análisis de sensibilidad de columna de destilación en la composición de glicerol de la corriente de proceso

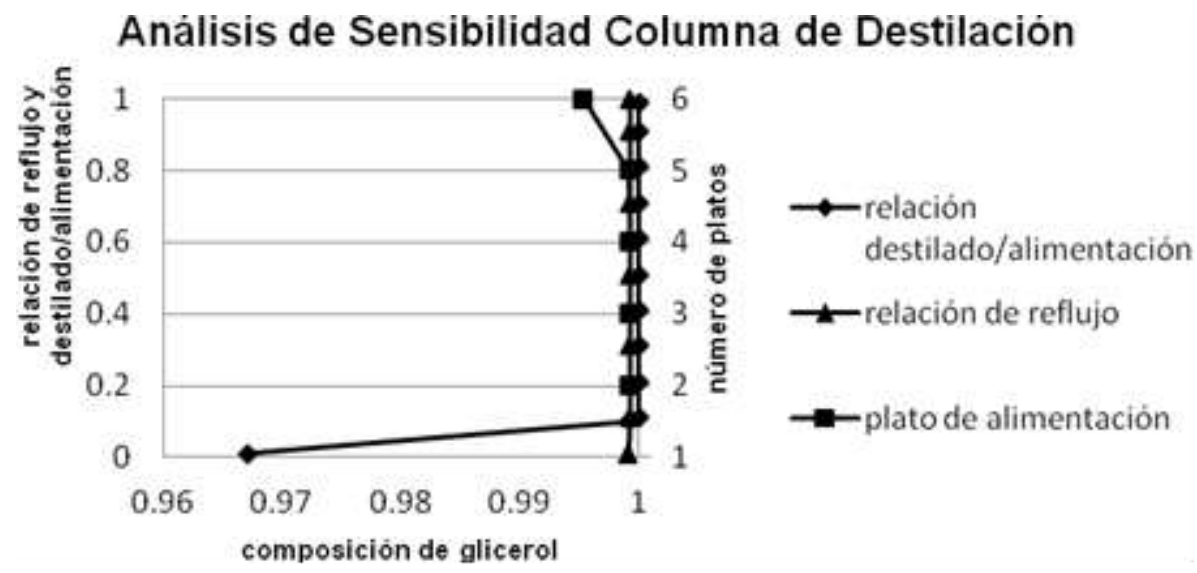

Previamente se determinó que para alcanzar la especificación de pureza requerida por la siguiente etapa del proceso (99\%) son necesario 6 de etapas de equilibrio en la mencionada columna de destilación. Se puede observar que tanto el caudal como la composición en la corriente de salida del producto deseado en este proceso son más sensibles a variaciones en la relación alimentación/destilado, mientras que una variación en el reflujo o en el plato el cual la columna es alimentada, no generan un cambio significativo en las especificaciones del glicerol purificado.

Figura 5 - Análisis de sensibilidad de columna de destilación en el caudal de glicerol de la corriente de proceso

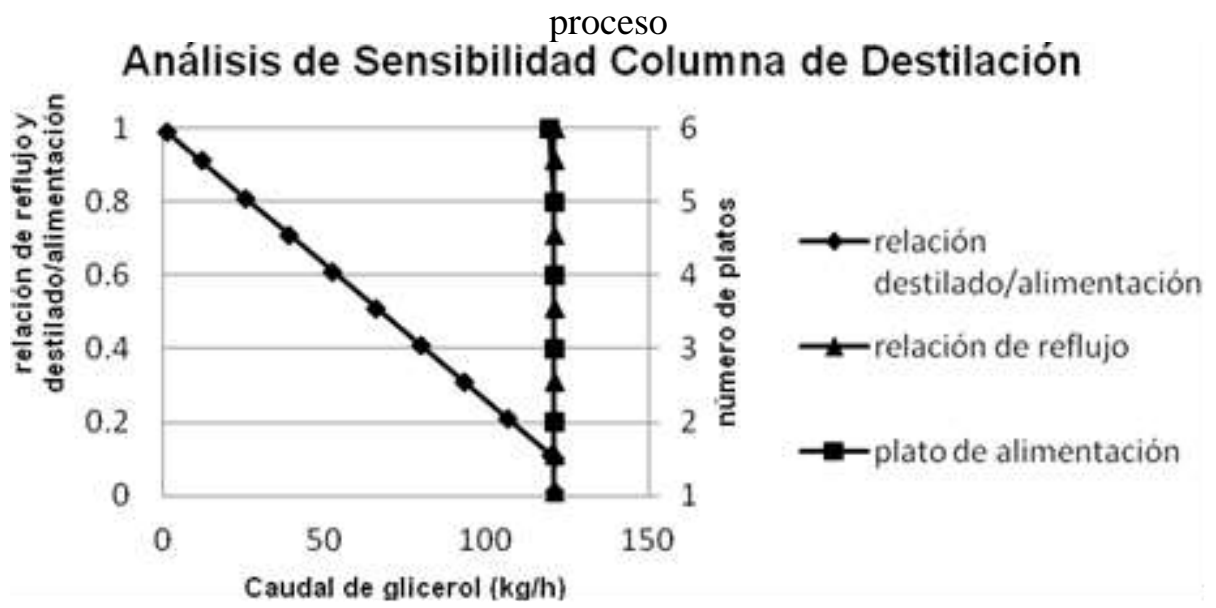

Los resultados de la optimización del proceso se muestran en las Tablas 1 a 3. 
Tabla 1 - Optimización de los intercambiadores de calor en la etapa de purificación de glicerol

\begin{tabular}{cccc}
\hline Equipos & $\begin{array}{c}\text { Presión de líquido } \\
\text { calefactor/refrigerante }\end{array}$ & $\begin{array}{c}\text { Temperatura del líquido } \\
\text { calefactor/refrigerante }\end{array}$ & $\begin{array}{c}\text { Caudal de líquido } \\
\text { refrigerante/calefactor }\end{array}$ \\
\hline $\mathrm{HE} 1$ & $1,70 \mathrm{bar}$ & $115^{\circ} \mathrm{C}$ & $50 \mathrm{~kg} / \mathrm{h}$ \\
$\mathrm{HE} 2$ & $1 \mathrm{bar}$ & $25^{\circ} \mathrm{C}$ & $81 \mathrm{~kg} / \mathrm{h}$ \\
$\mathrm{HE} 3$ & $1 \mathrm{bar}$ & $10^{\circ} \mathrm{C}$ & $84 \mathrm{~kg} / \mathrm{h}$ \\
$\mathrm{HE} 4$ & $5 \mathrm{bar}$ & $151,90^{\circ} \mathrm{C}$ & $24 \mathrm{~kg} / \mathrm{h}$ \\
\hline
\end{tabular}

Se reportan los valores óptimos para las corrientes auxiliares en los intercambiadores de calor a partir de las temperaturas óptimas de operación de cada uno de los flashes para lograr la remoción de los componentes no deseados en la corriente de proceso. Esto último se realiza utilizando el modo optimización de ASPEN, donde se define la función objetivo a minimizar, que para el caso de los intercambiadores de calor, resulta ser el caudal de la corriente auxiliar que debe ser provista a cada uno de ellos.

Tabla 2 - Optimización de los evaporadores en la etapa de purificación de glicerol

\begin{tabular}{ccc}
\hline Equipos & Temperatura de operación & Presión de operación \\
\hline FH1 & $112,9^{\circ} \mathrm{C}$ & $1 \mathrm{bar}$ \\
$\mathrm{FH} 2$ & $129,9^{\circ} \mathrm{C}$ & $1 \mathrm{bar}$ \\
\hline
\end{tabular}

Las restricciones a las que se encuentra sujeto este problema de optimización son las temperaturas requeridas de la corriente de proceso por los equipos aguas abajo del mismo (flashes, reactor y decantador). Se presenta también la relación alimentación/destilado de la columna de destilación de purificación de glicerol y la pureza requerida para ser utilizado como fuente de carbono en el proceso de fermentación.

Tabla 3 - Optimización de la columna de destilación en la etapa de purificación de glicerol

\begin{tabular}{ccccc}
\hline Equipos & $\begin{array}{c}\text { Número de etapas de } \\
\text { equilibrio }\end{array}$ & $\begin{array}{c}\text { Relación de } \\
\text { reflujo }\end{array}$ & $\begin{array}{c}\text { Relación de } \\
\text { alimentación/destilado }\end{array}$ & $\begin{array}{c}\text { Plato de } \\
\text { alimentación }\end{array}$ \\
\hline $\mathrm{CD}$ & 6 & 0,4 & 0,073 & 3 \\
\hline
\end{tabular}

Asimismo, se efectúa el dimensionamiento de los equipos que componen la planta de obtención de PHB, en el mismo entorno de optimización, con un factor de sobredimensionamiento de 1.4 (RAMOS et al., 2013). Esto último, permitirá en un futuro trabajo la posibilidad de una evaluación de costos operativos, como así también una maximización de los beneficios obtenidos en este proceso. Las Tablas 4 a 6 muestran los valores de las dimensiones de los equipos utilizados en las tres etapas del proceso: purificación de glicerol, fermentación y extracción y purificación de PHB (ULRICH, 1992). 
Tabla 4 - Dimensiones de equipos del sector de purificación de glicerol

\begin{tabular}{cccc}
\hline Equipos & Diámetro(m) & Altura o Largo $(\mathbf{m})$ & Área de intercambio $\left(\mathbf{m}^{\mathbf{2}}\right)$ \\
\hline Reactor & 0,50 & 1 & \\
Centrífuga & 0,11 & 0,47 & \\
Columna de destilación & 0,14 & & \\
Evaporadores & 0,26 & & \\
& 0,26 & 0,93 & 0,54 \\
Decantador & 0,31 & & 0,13 \\
Intercambiadores de calor & & & 0,23 \\
& & & 0,47 \\
Tanque de agua & 3,60 & 7,20 & \\
Tanque de glicerol & 2,38 & 4,75 & \\
Tanque de HCl & 0,64 & 1,28 & \\
\hline
\end{tabular}

En la Tabla 4 se observan las dimensiones características de los equipos que componen el primer sector de la planta de producción de PHB. Aquí se destaca principalmente la diferencia que existe entre las dimensiones de los equipos que actúan de manera directa en el proceso de purificación (reactor, centrífuga, evaporadores, etc.) respecto de los tanques que se utilizan como almacenamiento de producto (glicerol) y agua de servicio del proceso. Esto último se debe esencialmente a la significativa cantidad de agua de enfriamientocalentamiento que requiere esta etapa y al sobredimensionamiento correspondientemente utilizado debido a que se trata de tanques de almacenamiento.

Tabla 5 - Dimensiones de equipos del sector de fermentación

\begin{tabular}{cccc}
\hline Equipos & Diámetro(m) & Altura o largo $(\mathbf{m})$ & Área de intercambio $\left(\mathbf{m}^{2}\right)$ \\
\hline Fermentadores & 2,05 & 4,11 & \\
& 2,10 & 4,2 & 7,57 \\
Intercambiadores & & & 16,92 \\
de calor & & & \\
& & 9,28 & \\
Tanque de agua & 4,64 &
\end{tabular}

Tabla 6 - Dimensiones de equipos del sector de extracción de PHB

\begin{tabular}{cccc}
\hline Equipos & Diámetro(m) & Altura o Largo $(\mathbf{m})$ & Área de intercambio $\left(\mathbf{m}^{2}\right)$ \\
\hline Digestor & 0,55 & 1,1 & \\
Evaporador & 0,53 & 0,93 & 3 \\
Centrífuga & 0,22 & & 25 \\
Intercambiadores de calor & & & \\
& & 7,08 & \\
Tanque de solvente & 3,54 & 2,51 & \\
Tanque de PHB & 1,26 & 7,4 & \\
Tanque de agua & 3,70 & & \\
\hline
\end{tabular}

En los sectores de fermentación y extracción de PHB (Tablas 5 y 6), al igual que en el sector de purificación de glicerol, se puede distinguir la repercusión que tiene la cantidad de 
agua de servicio necesaria sobre las dimensiones características del tanque de almacenamiento de la misma.

Por otra parte, en la última etapa del proceso, la necesidad de solvente para la ruptura de la pared celular de los microorganismos en proporciones relativamente elevadas, demanda un dimensionamiento del tanque que lo almacena similar al tanque de agua de servicios auxiliares.

Finalmente, puede observarse que el área requerida por los intercambiadores de calor en estos sectores es sustancialmente mayor en comparación con el sector de purificación de glicerol. Esto último, se relaciona principalmente con el mayor caudal de proceso involucrado en los sectores de fermentación y extracción de PHB en comparación al de purificación de glicerol.

\title{
5 CONCLUSIONES
}

En el presente trabajo se ha llevado a cabo el modelado, análisis de sensibilidad y optimización de una planta de producción de Polihidroxialcanoatos mediante fermentación y usando glicerol (el co-producto de la producción de biodiesel) como sustrato.

El proceso es de creciente interés para la producción de biopolímeros, en particular empleando materias primas que no compitan con la alimentación.

El modelo se ha implementado en el simulador comercial Aspen Plus y se ha modificado para luego trabajar en modo optimización. Asimismo, se han dimensionado los equipos involucrados en el proceso.

Los resultados obtenidos a partir de los estudios de sensibilidad realizados brindan un aporte indispensable en el momento de reducir las numerosas variables de optimización que existen en equipos complejos, como se describe para la torre de destilación.

El modelo permite también la inclusión de las correlaciones de costos de capital, para el siguiente paso de maximización de los beneficios.

\section{OPTIMIZATION OF THE POLY( $\beta$-HYDROXYBUTYRATE) PRODUCTION FROM GLYCEROL}

\begin{abstract}
In this work, we carry out a preliminary design of a poly( $\beta$-hydroxybutyrate) (PHB) production plant employing microorganisms and glycerol as raw material. The process
\end{abstract}


consists of three main sectors: raw material purification, where the glycerol stream (byproduct of the biodiesel industry) is purified by eliminating methanol; fermentation, where PHB is produced from a strain of Cupriavidus necator; PHB extraction, where cellular walls are exposed to lyses and a purified product is obtained.

Keywords: Cupriavidus necator. Optimization. Poly( $\beta$-hydroxybutyrate).

\section{REFERENCIAS}

ASPEN PLUS VERSION 7.2. Aspen Technology, Inc., Cambridge, Massachusetts, USA, 2010.

CÁMARA ARGENTINA DE RECURSOS RENOVABLES. Disponible en: <http://www.cader.org.ar/informes-y-estudios/informes-energia-biodiesel-anosanterioers.htm $>$.

POSADA J.A.; CARDONA C.A.; RINCÓN L.E. Sustainable biodiesel production from palm using in situ produced glycerol and biomass for raw bioethanol. Proceedings of the 32nd Symposium on Biotechnology for Fuels and Chemicals, Clearwater Beach, Florida, April 19-22, 2010.

POSADA J.A.; NARANJO J.M.; LOPÉZ J.A.; HIGUITA J.C.; CARDONA C.A. Design and analysis of poly-3-hydroxybutyrate production processes from crude glycerol. Process Biochemistry, v. 46, n. 1, p. 310-317, 2011.

RAMOS, F.D.; ESTRADA, V.; DIAZ, M.S.; VILLAR, M.A.; RAMOS, F.D.; ESTRADA, V.; DIAZ, M.S.; VILLAR, M.A. Producción de Poli( $\beta$-hidroxibutirato) a partir de Glicerol. Simulación y Dimensionamiento de Equipos. Proceedings del X Simposio Argentino de Polímeros, Buenos Aires, Argentina, Agosto28-30, 2013.

ULRICH, G.D. Economía de los procesos de ingeniería química. McGraw-Hill 1992.

Originais recebidos em: 26/10/2013

Aceito para publicação em: 15/04/2014 